\title{
OPM magnetorelaxometry in the presence of a DC bias field
}

\author{
Aaron Jaufenthaler ${ }^{*}$ (D), Volkmar Schultze ${ }^{2}$, Theo Scholtes ${ }^{2}$ (D) Christian B. Schmidt ${ }^{2}$ (D), Michael Handler ${ }^{1}$, \\ Ronny Stolz ${ }^{2}$ (D) and Daniel Baumgarten ${ }^{1,3}$ (D)
}

\author{
"Correspondence: \\ aaron.jaufenthaler@umit.at \\ ${ }^{1}$ Institute of Electrical and \\ Biomedical Engineering, \\ UMIT_-Private University for Health \\ Sciences, Medical Informatics and \\ Technology, \\ Eduard-Wallnöfer-Zentrum 1, 6060 \\ Hall in Tirol, Austria \\ Full list of author information is \\ available at the end of the article
}

\begin{abstract}
Spatial quantitative information about magnetic nanoparticle (MNP) distributions is a prerequisite for biomedical applications like magnetic hyperthermia and magnetic drug targeting. This information can be gathered by means of magnetorelaxometry (MRX) imaging, where the relaxation of previously aligned MNP's magnetic moments is measured by sensitive magnetometers and an inverse problem is solved. To remove or minimize the magnetic shielding in which MRX imaging is carried out today, the knowledge of the influence of background magnetic fields on the MNP's relaxation is a prerequisite. We show MRX measurements using an intensity-modulated optically pumped magnetometer (OPM) in background magnetic fields of up to $100 \mu \mathrm{T}$. We show that the relaxation parameters alter or may be intentionally altered significantly by applying static fields parallel or antiparallel to the MNP's alignment direction. Further, not only the relaxation process of the MNP's magnetic moments could be measured with OPM, but also their alignment due to the MRX excitation field.
\end{abstract}

Keywords: Magnetic nanoparticles; Optically pumped magnetometers; Intensity-modulated OPM; Magnetorelaxometry; Bias field

\section{Introduction}

Magnetic nanoparticles (MNP) offer a variety of applications in biomedicine, e.g. magnetic hyperthermia [1] and magnetic drug targeting [2]. All these applications require knowledge about the quantitative spatial distribution of MNP and magnetorelaxometry (MRX) is a promising technique for their quantitative imaging. In MRX, the magnetic moments of the MNP are aligned by an external magnetic field, forming a net magnetic moment. After switching-off the external field, the MNP's net magnetic moment decays, which can be measured by highly sensitive quantum magnetometers, e.g. superconducting quantum interference devices (SQUID) [3] and optically pumped magnetometers (OPM) [4-6], as well as fluxgates [7]. Whereas the relaxation amplitude contains quantitative MNP information, temporal properties of the relaxation curves can be exploited to estimate, e.g., the binding state of MNP [8] or their temperature [9]. MRX with spatially different excitation fields allows, after solving an appropriate inverse problem, to obtain quantitative spatial MNP information, e.g. with SQUID [10] or commercially available OPM [11].

(c) The Author(s) 2020. This article is licensed under a Creative Commons Attribution 4.0 International License, which permits use, sharing, adaptation, distribution and reproduction in any medium or format, as long as you give appropriate credit to the original author(s) and the source, provide a link to the Creative Commons licence, and indicate if changes were made. The images or other third party material in this article are included in the article's Creative Commons licence, unless indicated otherwise in a credit line to the material. If material is not included in the article's Creative Commons licence and your intended use is not permitted by statutory regulation or exceeds the permitted use, you will need to obtain permission directly from the copyright holder. To view a copy of this licence, visit http://creativecommons.org/licenses/by/4.0/. 
Currently, MRX imaging is performed within magnetic shieldings and thus at low background magnetic fields $(<\mu \mathrm{T})$, although MNP quantification with MRX has been shown in noisy environments using SQUID gradiometers [12] and fluxgate gradiometers [7]. In Dolgovskiy et al. [13], OPM-MRX is performed within a weak magnetic shield. Novel OPM employing miniaturized vapor cells $[14,15]$ are beneficial for magnetorelaxometry imaging measurements due to their small sensing volume and the possibility of flexible positioning. Moreover, these sensors have been shown to potentially reach sensitivities down to tens of $\mathrm{fT} / \sqrt{\mathrm{Hz}}$, even when operated within fields of geomagnetic strength (tens of $\mu \mathrm{T}$ ) [16-18]. As magnetic shieldings are a crucial factor in terms of costs, availability and flexibility, it is desirable to avoid or minimize shielding in clinical applications. Unshielded magnetic particle spectroscopy (MPS), AC susceptometry (ACS) and magnetic particle imaging (MPI) with OPM were already shown [19, 20]. Besides solving arising problems with environmental noise, a detailed understanding of how background magnetic fields influence the MNP relaxation processes is a prerequisite. It has been shown experimentally using SQUID, that Néel relaxation can be accelerated by applying external magnetic fields [21]. Transverse MRX of MNP wearing a viscoelastic corona, with an applied background magnetic field perpendicular to the excitation field has been modeled and investigated mathematically. It was shown in simulations that in transverse MRX, background magnetic fields alter the shape of the relaxation and decrease the relaxation time constant [22]. The dynamics of MNP's magnetic moments can be described by two concurrent processes: rotation of the whole particle is named Brownian motion, whereas the flipping of the single MNP's magnetic moments along their easy axis is described by so-called Néel relaxation [23]. As modeled mathematically by Fokker-Plank equations, it has been shown that the Brownian and Néel relaxation time constants decrease monotonically with increasing magnetic field strength, while for large fields $(>\mathrm{mT})$ Néel relaxation is much more sensitive to magnetic fields [24]. Further, the dependence of the Néel relaxation time $\tau_{\mathrm{N}}$ on an applied field is well known $[25,26]$ :

$$
\tau_{\mathrm{N}}(H)=\tau_{0} \cdot \exp (1-0.82 h)
$$

where $\tau_{0}$ is a constant and $h=\frac{H}{H_{\mathrm{k}}}$, where $H$ denotes the applied (background) field and $H_{\mathrm{k}}$ the anisotropy field. Therefore, the Néel relaxation time decreases with an increase of the applied magnetic field.

In this paper, we present an OPM-MRX measurement setup for measuring within different defined background magnetic fields, ranging from $5 \mu \mathrm{T}$ to $100 \mu \mathrm{T}$, and analyze the obtained data. We show, that the background magnetic field strongly influences MNP relaxation parameters. The relaxation parameters not only depend on the background magnetic field amplitude, but also on its direction. Finally, we show proof of principle measurements, where we detect not only the relaxation of MNP's magnetic moments, but also their alignment by the excitation field.

\section{Methods}

\subsection{Setup overview}

Our setup consists of a single channel OPM, an excitation coil for MNP alignment and a 3D Helmholtz coil system for the generation of defined background magnetic fields. The setup is positioned within a three-layer magnetic shielding barrel [27]. A MNP sample is placed on top of the OPM vapor cell, surrounded by the excitation coil (Fig. 1). 
Figure 1 Setup overview. MRX setup with OPM, MNP sample and MNP excitation coil (blue)

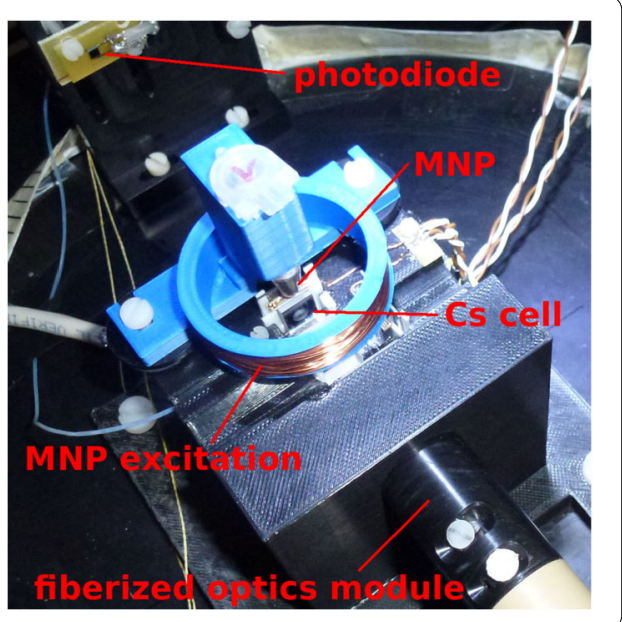

Figure 2 Simplified schematic of coil driver: constant current source 11 , high voltage MOSFET M1, TVS diode D1 and the coil for MNP excitation, modeled as RL-circuit (R1, L1)

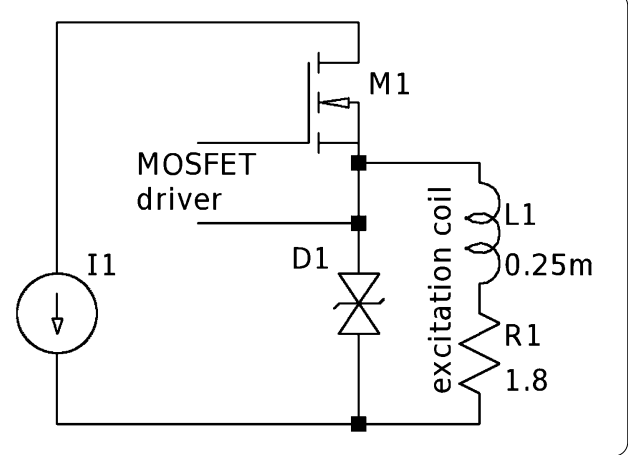

\subsection{MNP excitation circuit}

The MNP's magnetic moments are aligned by a magnetic field of about $1 \mathrm{mT}$ (unless otherwise noted), generated by a 65-turn, $48 \mathrm{~mm}$ diameter coil (Fig. 1). After $200 \mathrm{~ms}$ (unless otherwise noted), this excitation field is shut-off within a few $\mu \mathrm{s}$, which is achieved by a fast de-energizing of the coil. This is ensured by a low coil inductance and a fast-switching high-voltage MOSFET, combined with a high-voltage rated transient-voltage-suppression (TVS) diode (Fig. 2). The TVS diode clamps the back EMF to a (maximum) fixed level, protecting the MOSFET and decreasing the magnetic field linearly over time.

\subsection{Intensity-modulated OPM}

A schematic overview of the OPM is shown in Fig. 3. The OPM employs a microfabricated Cs vapor cell with nitrogen buffer gas (38 mbar) and a sensing volume of approx. $50 \mathrm{~mm}^{3}$. The cell consists of a $4 \mathrm{~mm}$ thick silicon body with a cylindrical cavity, closed by anodically bonded glass faces [28]. The vapor cell is operated at $70^{\circ} \mathrm{C}$ by electrical thin-film heaters glued to the side faces of the Si body and driven by a $10 \mathrm{kHz}$ ac current. Circularly polarized light from a distributed Bragg reflector (DBR) laser diode at the Cs D1 transition $(895 \mathrm{~nm})$ is intensity-modulated at the chopping frequency $f_{\mathrm{c}}$ resonantly tuned to the Larmor frequency $f_{\mathrm{L}}$ of the Cs atoms. It should be noted that $f_{\mathrm{c}}$ is not feedback-controlled to avoid an increased settling time and transient signals after switching-off the MNP's excitation field. In this all-optical Bell-Bloom configuration, the magnetometer sensitivity is optimized when operated in static magnetic fields oriented perpendicular to the laser 


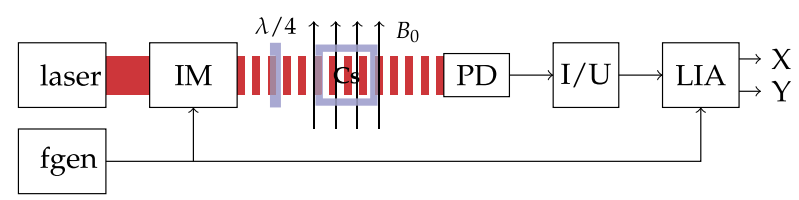

Figure 3 Schematic of the OPM configuration. With a function generator (fgen) and intensity modulator (IM), the linearly polarized light from the laser is pulsed at the Larmor frequency of the alkali atoms and converted into circular polarization by a $\lambda / 4$ waveplate. The transmitted laser light is detected by a photodiode (PD), amplified by a current voltage converter with integrated preamplifier $(I / U)$ and phase-sensitively demodulated with a lock-in amplifier (LIA)

beam propagation direction $[29,30]$. The laser light power transmitted through the vapor cell is detected by a Si photodiode.

\subsection{Data acquisition and preprocessing}

The OPM photodiode signal is passed through a current voltage converter $(\mathrm{I} / \mathrm{U})$ and amplifier. Then, the signal is mixed with the OPM laser chopping frequency $f_{\mathrm{c}}$ by the lock-in amplifier (LIA), and the resulting in-phase component (LIA-X) and quadrature component (LIA-Y) are directly digitized by the LIA after 4th order low-pass filtering with a $-3 \mathrm{~dB}$ bandwidth of $10 \mathrm{kHz}$. A sample rate of $107.1 \mathrm{kHz}$ is used. Additionally, the MNP excitation coil current is recorded by the same device, which serves as trigger for the data processing. In order to remove spurious frequency components emerging from the electrical ac heating, the OPM data is preprocessed in software by low-pass filtering with a cutoff-frequency of $1 \mathrm{kHz}$ for liquid MNP and $100 \mathrm{~Hz}$ for MNP immobilized in gypsum. The digital filter is realized as bessel filter to preserve sharp edges in the data.

\subsection{MNP and MRX model}

Magnetic nanoparticles with a hydrodynamic diameter of $100 \mathrm{~nm}$ (plain BNF-Dextran) from Micromod (Micromod Partikeltechnologie GmbH, Rostock, Germany) were used in this experiment. The MNP are dextran based, water suspended and their iron-oxide core with a diameter of $45 \mathrm{~nm}$ consists of multiple 15-20 nm iron-oxide crystallites [31]. Two samples of $140 \mu \mathrm{l}$ were prepared for the experiment. One sample is composed of $100 \mu \mathrm{l}$ liquid (factory supplied) MNP and diluted in $40 \mu \mathrm{l}$ distilled water, whereas the second sample consists of $100 \mu \mathrm{l}$ MNP, embedded and thus immobilized in gypsum, resulting in a total sample volume of $140 \mu \mathrm{l}$. The undiluted iron concentration is about $15 \mathrm{mg} / \mathrm{ml}$, resulting in an iron amount of $1.5 \mathrm{mg}$ in each sample.

The MNP relaxation signal $B(t)$ can be described in several ways. For an MNP system with uniform particle size and non-interacting particles, a single exponential decay of the associated magnetic flux density at the OPM's location is expected [32]:

$$
B_{\text {ideal }}(t)=B_{\mathrm{R}} \exp \left(-\frac{t}{\tau_{\mathrm{R}}}\right)
$$

with the relaxation amplitude $B_{\mathrm{R}}$, the relaxation time constant $\tau_{\mathrm{R}}$ and the time $t$ after the start of the relaxation. However, real MNP systems usually show a non-uniform particle size distribution. Assuming a magnetometer bandwidth of $500 \mathrm{~Hz}$ and a typical MNP anisotropy of $10^{4} \mathrm{~J} / \mathrm{m}^{3}$, particles with a core diameter in the interval [17.5 nm, $21 \mathrm{~nm}$ ], 
while having a hydrodynamic diameter $\geq 100 \mathrm{~nm}$, contribute to the MRX signal and therefore can be detected [2]. For MNP ensembles with an equally distributed diameter in the detectable size range, the relaxation can be described by $[3,26]$ :

$$
B_{\text {unif }}(t)=B_{\mathrm{R}} \ln \left(1+\frac{\tau}{t}\right)
$$

with the amplitude $B_{\mathrm{R}}$ and a time constant $\tau$ which depends on the excitation parameters and the MNP's anisotropy. The relaxation signal can also be described phenomenologically by a stretched exponential [32]:

$$
B_{\mathrm{ph}}(t)=B_{\mathrm{R}} \exp \left[-\left(\frac{t}{\tau_{\mathrm{R}}}\right)^{\beta}\right]+B_{\text {offset }}
$$

with the relaxation amplitude $B_{\mathrm{R}}$, the relaxation time constant $\tau_{\mathrm{R}}$ and the stretching parameter $\beta$. The offset $B_{\text {offset }}$ is introduced to compensate for magnetometer heading error, background magnetic fields and static MNP magnetization (e.g. due to the applied background magnetic field).

In immobilized MNP, Brownian motion is suppressed, while in water suspended samples both, Brownian motion and Néel motion may contribute to the MRX signal. Therefore, it reasonable to fit for both fractions [32]:

$$
B(t)=B_{\mathrm{b}}+B_{\mathrm{ub}}+B_{\text {offset }},
$$

with the contribution of bound (immobilized) MNP $B_{\mathrm{b}}$ and unbound MNP $B_{\mathrm{ub}}$, where for each, one or a linear combination of the previously presented approaches may be well suited.

When fitting the measured data to such a model, the high number of parameters leads to an ill-conditioned problem. Even for simple models, the parameter variance and mutual interdependence is often very high, not only due to the contribution of environmental noise. To overcome this uncertainty, first a model is fitted to the experimental data and the absolute amplitude difference at two fixed time points is used as measure for the relaxation amplitude.

A robust parameter for the relaxation time is the integral relaxation time [33], often called correlation time [34]. It is denoted as the area under the amplitude-normalized relaxation curve. For the estimation of this parameter no curve-fitting was involved.

\subsection{MNP relaxation data processing}

Since the relaxation signal is of an exponential form, a high OPM bandwidth is required to capture early parts of the relaxation. Latter parts of the relaxation, however, do not require high bandwidths, but would benefit from a high magnetometer sensitivity. To satisfy both requirements, adaptive filtering or resampling can be implemented [32]. With a similar effect, in this work, the data is weighted exponentially during curve fitting. The fits of the data to the relaxation model, which was selected as the sum (5) of two stretched exponentials (4), were performed using the trust-region-reflective least squares algorithm [35] provided by Matlab ${ }^{\circ}$. For the extraction of the relaxation amplitude and the integral relaxation 
time, time intervals are selected as [12 ms, $280 \mathrm{~ms}$ ] for liquid MNP and [0.12 s, $6.5 \mathrm{~s}$ ] for immobilized MNP, respectively. This data analysis is repeated for several subsequent MRX sequences at a fixed background magnetic field. Additionally, the analysis is performed on three or seven times averaged MRX data for immobilized or liquid MNP, respectively.

\section{Experimental results and discussion}

\subsection{OPM characterization}

Figure 4 shows the noise level of the OPM at different background magnetic fields, which was varied from $5 \mu \mathrm{T}$ to $100 \mu \mathrm{T}$. The noise floor was around $500 \mathrm{fT} / \sqrt{\mathrm{Hz}}$ at $500 \mathrm{~Hz}$ for all configurations, limited by excess laser intensity noise, while the shot-noise limited OPM sensitivity level was $51 \mathrm{fT} / \sqrt{\mathrm{Hz}}$. In previous detailed studies of a similar setup we were able to suppress excess intensity noise by subtraction of a reference photo diode signal by a factor of 3 [30]. Common visible spikes in the noise spectrum arise at the mains frequency and its harmonics, whereas the spikes occuring at a single background magnetic field only, e.g., the spike at $390 \mathrm{~Hz}$ at $B_{0}=5 \mu \mathrm{T}$, arise from LIA mixing (leakage) with the ac current driving the OPM heater.

The OPM frequency response was estimated using a spectrum analyzer (HP 35460A) and a random-noise-fed pancake coil, placed near the OPM (Fig. 5). The OPM bandwidth was estimated to be about $500 \mathrm{~Hz}$. It is noted, that the bandwidth does not significantly change at different background magnetic fields $\leq 100 \mu \mathrm{T}$.

Next, the region of linear OPM response was estimated. At a fixed background magnetic field $B_{0}$, and thus at a fixed Larmor frequency $f_{\mathrm{L}}$, the OPM laser chopping frequency was swept over a range of $f_{\mathrm{L}} \pm 3.5 \mathrm{kHz}$ corresponding to $B_{0} \pm 1 \mu \mathrm{T}$. The raw LIA-Y and LIA-magnitude (LIA-R) data are depicted in Fig. 6. Each of the measured LIA-Y transfer functions was used to convert the LIA-output to an equivalent magnetic field. While the region of linear response depends on the background magnetic field, only sensor data within a region of $\pm 200 \mathrm{~Hz}$ or $\pm 57 \mathrm{nT}$, respectively, was used for the MNP relaxation fits, while still keeping in mind the sensor bandwidth.

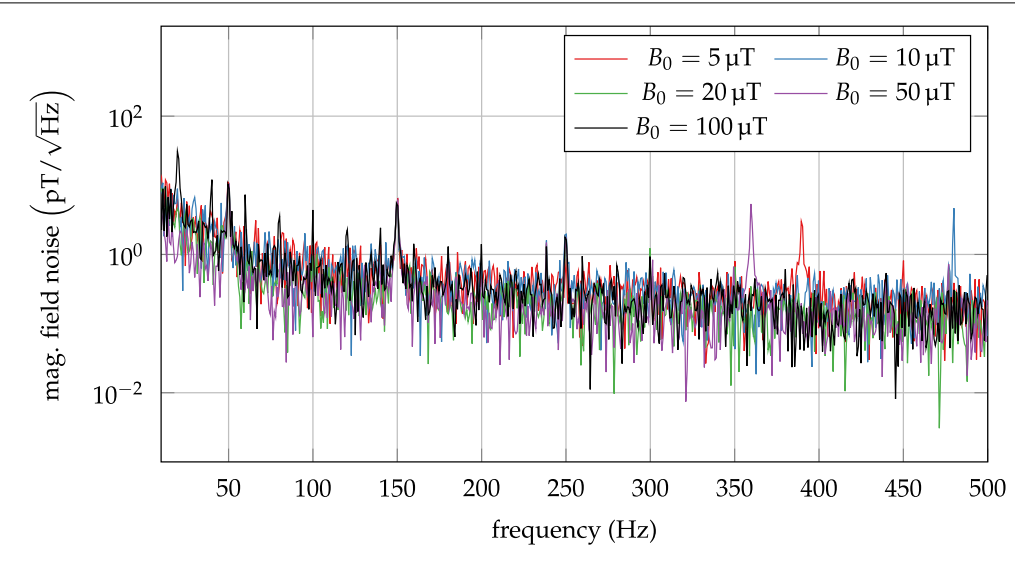

Figure 4 OPM noise spectrum. Base noise of the OPM at different background magnetic fields from $5 \mu T$ to $100 \mu T$, measured within a three layer magnetically shielded barrel 


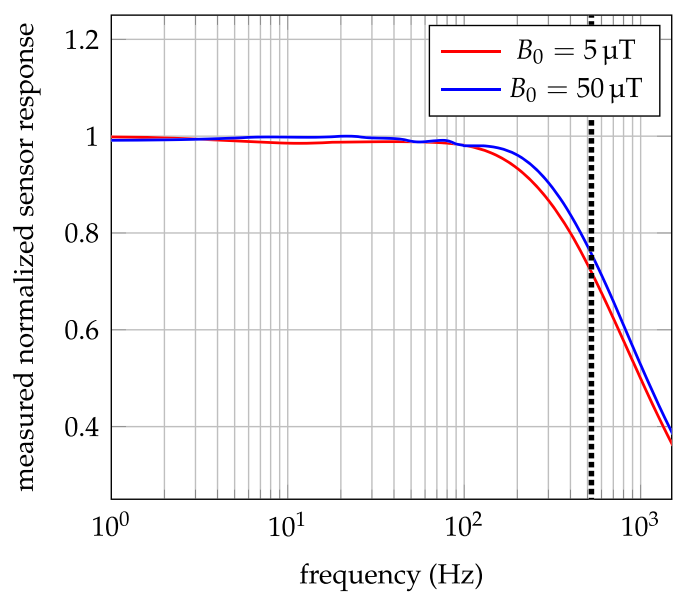

Figure 5 Measured OPM frequency response at $B_{0}=5 \mu \mathrm{T}$ and $B_{0}=50 \mu \mathrm{T}$. The dotted line indicates the $-3 \mathrm{~dB}$ bandwidth at $\approx 500 \mathrm{~Hz}$. The data was acquired using a spectrum analyzer and a random-noise-fed pancake coil

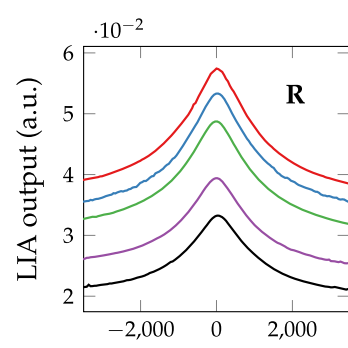

(a) frequency detuning $f_{\mathrm{L}}-f_{\mathrm{c}} \quad(\mathrm{Hz})$
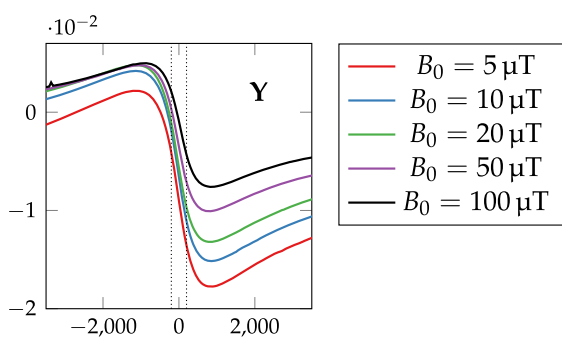

(b) frequency detuning $f_{\mathrm{L}}-f_{\mathrm{c}}(\mathrm{Hz})$

Figure 6 OPM resonances. Cesium resonances, acquired with the lock-in amplifier at different background magnetic fields. (a) Magnitude (LIA-R), (b) quadrature component (LIA-Y); The frequency axes are centered at the Larmor frequency. The region of linear Y-response used for data analysis is marked by black dotted lines

\subsection{Estimation of relaxation parameters: dependence of liquid MNP's relaxation behavior on background magnetic fields}

First, the sample of liquid $100 \mathrm{~nm}$ MNP was positioned in the system. Then, the excitation coil was pulsed $(200 \mathrm{~ms}, 1 \mathrm{mT})$ and the relaxation of the MNP was measured. This was repeated at different background magnetic field strengths. As it can be seen in Fig. 7(a), the relaxation curve is changing in dependence of the $B_{0}$ field modulus. Regarding the excitation pulse direction with respect to the $B_{0}$ field, a parallel and an antiparallel configuration was investigated. It should be noted that the direction of the $B_{0}$ field was kept constant during the whole experiment, but instead the excitation pulse direction was flipped. When investigating the raw OPM signals, care must be taken of the nonlinear OPM response and the limited OPM bandwidth. Therefore, for this configuration the first $10 \mathrm{~ms}$ of data were clipped. The estimated relaxation parameters are shown in Fig. 8. It can be seen that for both, parallel and antiparallel excitation, the relaxation amplitude decreases with an increase of the background magnetic field. In contrast, the integral relaxation time decreases for antiparallel background magnetic fields, whereas it increases for parallel background 

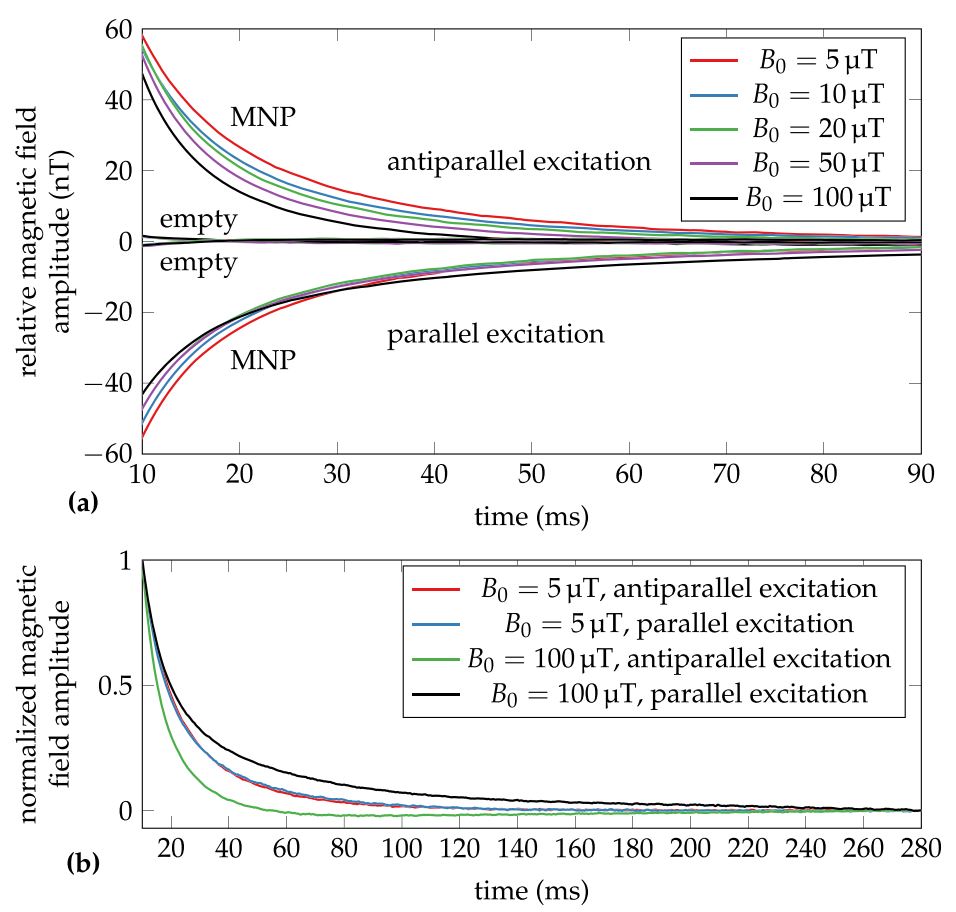

Figure 7 (a) MRX of liquid MNP with parallel and antiparallel excitation fields with respect to the background magnetic fields. The data was acquired with the OPM, 1 kHz low-pass filtered and seven-times averaged. (b) Amplitude-normalized relaxation signals
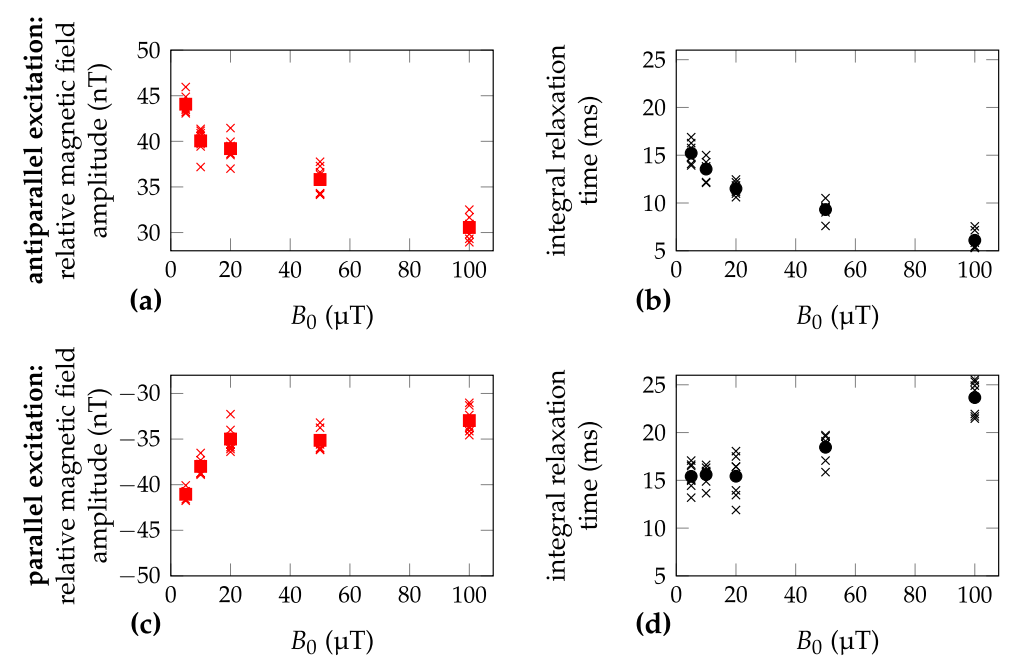

Figure 8 Relaxation amplitudes and integral relaxation time constants of liquid MNP at different background magnetic fields. Antiparallel excitation (a), (b) and parallel excitation (c), (d). Crosses indicate results for unaveraged data, squares and dots indicate results for seven-times averaged data

magnetic fields. To emphasize the different relaxation-dynamics for parallel and antiparallel excitation, a selected set of amplitude-normalized relaxation signals is depicted in Fig. 7(b). 


\subsection{Estimation of relaxation parameters: dependence of immobilized MNP's relaxation behavior on background magnetic fields}

The immobilized MNP sample was positioned in the system and the MNP relaxation was measured after pulsing the excitation coil. This was repeated at different background magnetic field strengths. Figure 9 shows data averaged from three measurement cycles and the estimated relaxation parameters are depicted in Fig. 10. It can be seen that the relaxation amplitude is decreased for parallel excitations, when increasing the background magnetic field. For antiparallel excitations, the amplitude varies only by a few percent. The integral relaxation time is increased and decreased for antiparallel and parallel excitations, respectively, when increasing the background field.

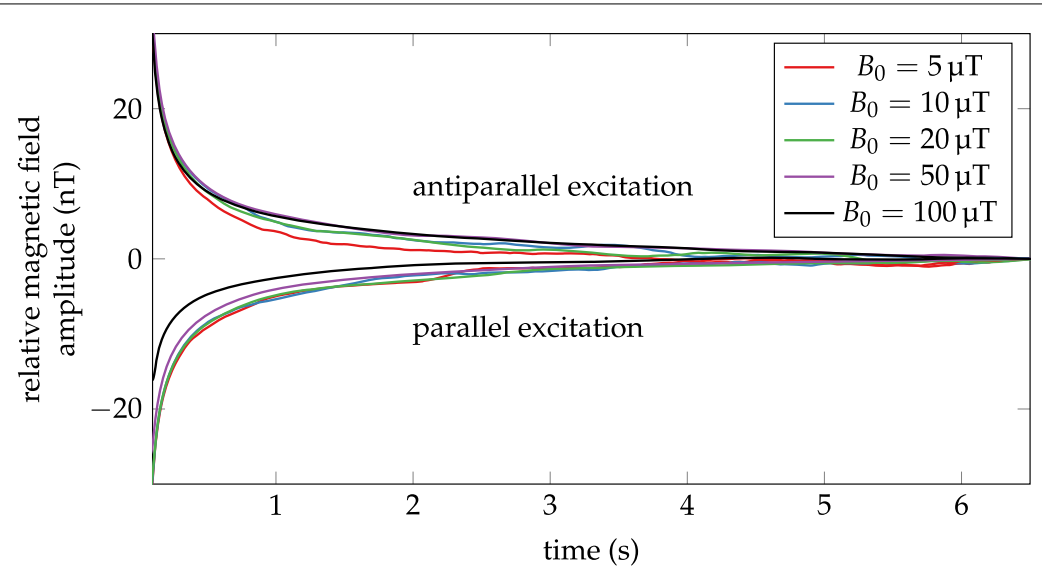

Figure 9 MRX of immobilized MNP at different background magnetic fields. The excitation field was applied parallel and antiparallel with respect to the background magnetic field. The data was acquired with the OPM, $100 \mathrm{~Hz}$ low-pass filtered and three-times averaged
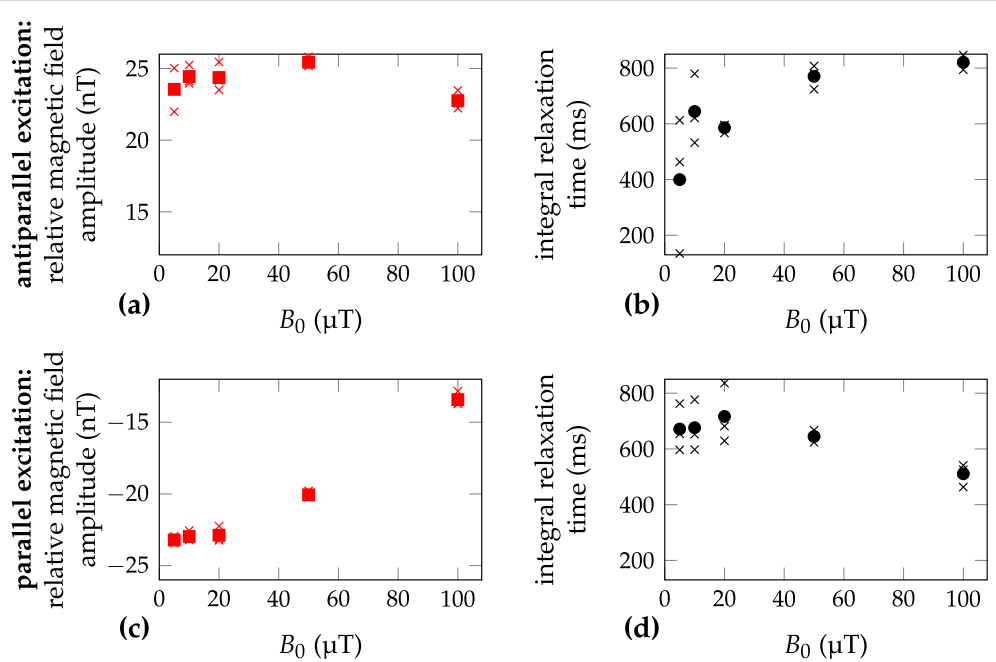

Figure 10 Relaxation amplitudes and integral relaxation time constants of immobilized MNP at different background magnetic fields. Antiparallel excitation (a), (b) and parallel excitation (c), (d). Crosses indicate results for unaveraged data, squares and dots indicate results for three-times averaged data 


\subsection{Proof of principle inverse MRX}

While MRX at different background magnetic fields was investigated, also the inverse process, namely the alignment of the MNP's magnetic moments with the excitation magnetic field may be of interest for various applications. Especially for MRX imaging, it is desired to measure both, the alignment and relaxation of the MNP's magnetic moments, to reduce the data acquisition time or to improve the ill-posed inverse problem.

Here, we turn on and off an external $65 \mu \mathrm{T}$ magnetic field periodically (at $0.1 \mathrm{~Hz}$ ), while a constant background field of $5 \mu \mathrm{T}$ is applied continuously to the system. The static field and the excitation field are applied antiparallel to each other. Thus, to track both processes (alignment and decay) simultaneously, it is desirable to have a magnetometer which is sensitive at both $5 \mu \mathrm{T}$ and $60 \mu \mathrm{T}$, without being limited by a possible dead time or small sensor slew rate during the stepwise magnetic field change. Our OPM usually has a dynamic range of approx. $60 \mathrm{nT}$ and so it cannot cover the whole range. To avoid this limitation, one possibility is to locally compensate the excitation field, e.g., using self-compensating solenoids [20]. However, the practical realization of field compensations might be tricky. A well known way of extending the OPM's dynamic range is to employ a feedback loop [36]. As stated above, we did not want to follow this approach as we wanted to avoid transient signals after switching-off the excitation field.

A novel approach, the "bi-chromatic intensity modulation", is presented: The center of the sensitive range of the sensor is set by the laser chopping (and thus lock-in amplifier) frequency $f_{\mathrm{c}}$, which corresponds to a Larmor frequency at a specific magnetic field amplitude. To obtain a sensitivity at both magnetic field levels, we apply a bichromatic signal to the intensity modulator, chopping the light at two frequencies corresponding to the $\mathrm{Cs}$ vapor Larmor frequency during alignment and decay of MNP's magnetic moments, respectively. We demodulate the photodiode signal with two time synchronized LIA at each individual reference frequency.

In Fig. 11 it can be seen that one of the LIA detects the relaxation, while the other one detects the alignment of the MNP's magnetic moments. Both signals are analyzed separately
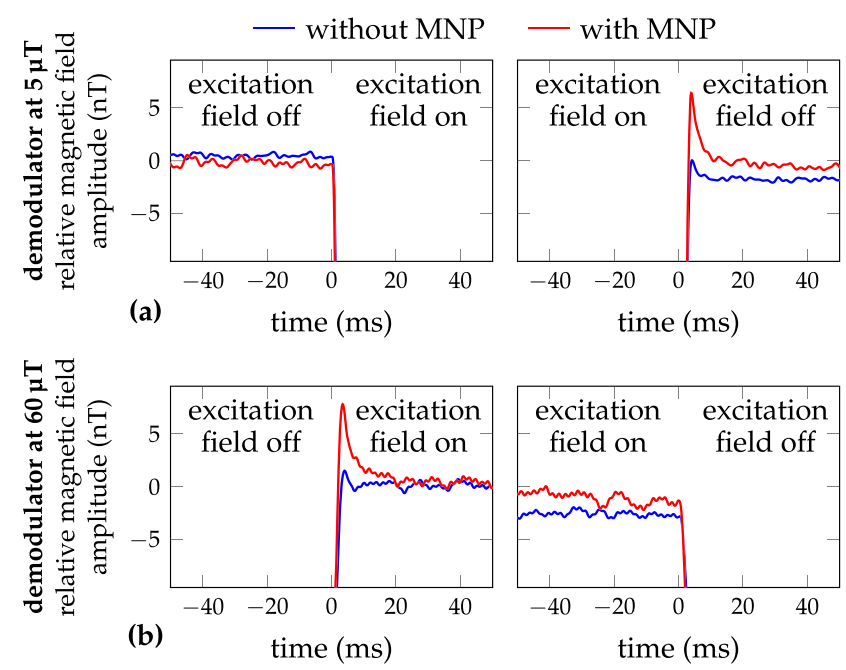

Figure 11 MRX and inverse MRX with bichromatic chopping and dual-demodulation OPM. Amplitude scaled raw data of LIA1 (a) at $18.8 \mathrm{kHz}$ and LIA2 (b) at $216.8 \mathrm{kHz}$. Empty measurement in blue, measurement with MNP in red. The data was not averaged 
in the time intervals ranging from $2 \mathrm{~ms}$ to $18 \mathrm{~ms}$. The relaxation amplitude and the integral relaxation time were estimated as $B_{\mathrm{R}}=3.7 \mathrm{nT}$ and $\mathrm{T}_{\mathrm{R}}=9.75 \mathrm{~ms}$, respectively, while the alignment amplitude and the integral alignment time were estimated as $B_{\mathrm{A}}=3.1 \mathrm{nT}$ and $\mathrm{T}_{\mathrm{A}}=8.56 \mathrm{~ms}$, respectively. Thus, the integration time constant and the amplitude decrease with increased magnetic field.

\section{Discussion}

We presented a novel experimental investigation of the influence of parallel and antiparallel background magnetic fields on the relaxation of MNP.

By increasing the antiparallel background magnetic field from $5 \mu \mathrm{T}$ to $100 \mu \mathrm{T}$, the integral relaxation time decreased by $60 \%$ (from $15.2 \mathrm{~ms}$ to $6.1 \mathrm{~ms}$ ) in our liquid MNP sample. In the parallel configuration, the integral relaxation time increased from $15.4 \mathrm{~ms}$ to $23.7 \mathrm{~ms}$, which corresponds to an increase of $54 \%$. This decrease of the integral relaxation time for antiparallel excitation and the increase of the integral relaxation time for parallel excitaton is in good agreement with the literature: As calculated by Deissler et al. [24], the magnetic energy density (for Néel and Brownian relaxation combined) of MNP for parallel fields decreases, while it increases for antiparallel fields, respectively. The magnetic energy density is proportional to the relaxation time [24].

Regarding the relaxation amplitude, three counteracting effects have to be considered. Firstly, the effectively applied excitation field is increased or decreased by the background magnetic field. It is well known that the relaxation amplitude increases linearly with an increase of the excitation field (for excitation fields up to several $\mathrm{mT}$ ) [37]. Secondly, due to the extraction of the relaxation amplitude at two fixed time points, this parameter is coupled with the integral relaxation time. Thirdly and finally, the background magnetic field, if applied parallel to the excitation field, prevents some MNP from relaxing. In our liquid MNP sample, the absolute value of the relaxation amplitude decreased by $31 \%$ or $20 \%$ by increasing the background magnetic field for antiparallel or parallel excitation, respectively.

For the MNP response in our immobilized sample, the partially non-monotonical response of the extracted parameters to the background magnetic field may be explained by considering the non-homogeneous size distribution in our sample. As Coffey et al. [38] emphasize, the influence of the parameter $h$ (cp. Eq. (1)) depends strongly on the energy barrier height of the Néel relaxation. Therefore, by increasing the background magnetic fields, the different size fractions in the MNP sample behave differently. According to the previously mentioned particle size effect study it is likely that hereby the MNP relaxation shape and as a consequence thereof the integral relaxation time changes.

The integral relaxation times of our liquid MNP sample are below $30 \mathrm{~ms}$ and the integral relaxation times of our immobilized MNP sample are in the region of several $100 \mathrm{~ms}$. While the immobilized sample is governed by Néel relaxation only, in the liquid sample both Néel and Brownian relaxation occur. In view of the different time scales for the two types of samples, one might be inclined to neglect Néel relaxation in the liquid samples. However, this is only reasonable if Brownian and Néel relaxation can be considered to be uncoupled phenomena, but different groups have shown that in fact they are not [24, 39]. As a consequence, modeling and experimental characterization practice has to take both effects into account in most cases. Improved understanding in mechanisms affecting 
Néel and Browian relaxation in different ways, like, e.g., orientation and strength of static magnetic fields, may help to disentangle intricate MNP dynamics. The importance of the interplay of the relaxation mechanisms is substantiated, e.g., by the study of Dolgovskiy et al. [13], where it has been shown that small variations in the MNP diameter distribution might have a big impact on the measurement results obtained by MRX.

The results from our proof of principle inverse MRX, where the integral time and amplitude decrease with increased magnetic field, are in accordance to the theory $[24,40]$ and our other measurement results for antiparallel excitation.

\section{Conclusion and outlook}

In conclusion, we demonstrated MRX of MNP at background fields up to $100 \mu \mathrm{T}$ using an intensity-modulated OPM. We have investigated experimentally, how the relaxation processes of liquid and immobilized MNP's magnetic moments alter or can be altered on purpose by applying static background magnetic fields in parallel and antiparallel to the excitation field. For biomedical applications it is particularly important to investigate and differentiate both liquid and immobilized MNP to obtain information about the environment of the particles [41]. In this respect, also intermediate states and environments will be of interest with the aim of a multicolor imaging [42]. The knowledge of the background magnetic field's effect on the relaxation is an important prerequisite for unshielded MRX and may lead to new spatial encoding schemes in MRX imaging, similar to [21]. In the future, background magnetic fields perpendicular to the excitation field will be investigated. Hand in hand, MNP model estimation studies with larger parameter variation in terms of background fields and excitation fields (amplitude, duration and direction) will be undertaken. The variation of excitation fields is especially important for unshielded MRX imaging or novel MRX imaging spatial encoding schemes. In unshielded MRX (imaging), the effect of the background magnetic field (e.g. Earth's magnetic field) has to be considered and modeled. The inverse problem in MRX imaging significantly benefits from inhomogeneous excitation fileds [43]. In a similar way, an additional spatial encoding scheme using (temporaly active) homogeneous or inhomogeneous background magnetic fields could potentially improve the imaging resolution and accuracy. When applying inhomogeneous background fields, the MNP sample's response will be a superposition of responses at various background magnetic fields, which have to be entangled in post-processing, e.g. using a multicolor approach [42].

In this paper we have shown that not only the relaxation, but also the alignment of MNP's magnetic moments can be measured with our intensity-modulated OPM. This additional information might decrease the critical data acquisition time, e.g., during a treatment, of MRX imaging by at least a factor of two. Furthermore, such an approach may open the way towards new MRX imaging modalities in the future, e.g. using stepwise excitation and relaxation schemes. For unshielded measurements, a feedback loop of the laser modulation frequency will be embedded to enhance the OPM bandwidth and measurement range $[36,44]$. The OPM sensitivity might be improved by adding a (magnetically shielded) reference cell [30] or by using multipass cells [45]. Obviously, a gradiometric OPM setup would facilitate background noise suppression in unshielded environments [46].

To sum up, we believe that given previous work achieving unshielded OPM measurements of biomagnetic signals (e.g. magnetoencephalography [46]) and the understanding 
of MNP's relaxation processes at different background magnetic fields, unshielded MRX (imaging) will be feasible. An unshielded setup will foster the impact and availability of MNP imaging, in particular with respect to monitoring of therapeutic applications such as magnetic hyperthermia and magnetic drug targeting.

\author{
Acknowledgements \\ We thank Victor Lebedev from PTB Berlin for temporary loan of a Femto DHPCA-100 amplifier and Gregor Oelsner from \\ Leibniz-IPHT for fruitful discussions and careful proofreading.

\section{Funding} \\ This research was funded by the German Science Foundation (DFG) within the priority program 1798 (grant \\ no. BA4858/2-1). The funding of the project 2017 FE 9128 by the Free State of Thuringia was co-financed by European \\ Union funds under the European regional Development Fund (ERDF).
}

\author{
Abbreviations \\ Back EMF, Back Electromotive Force; DBR, Distributed Bragg Reflector; LIA, Lock-In Amplifier; MNP, Magnetic \\ Nanoparticles; MOSFET, Metal-Oxide-Semiconductor Field-Effect Transistor; MRX, Magnetorelaxometry; OPM, Optically \\ Pumped Magnetometer; SQUID, Superconducting Quantum Interference Device.
}

\title{
Availability of data and materials
}

The data generated and analysed during the current study is available from the corresponding author on reasonable request.

\section{Competing interests}

The authors declare that they have no competing interests.

\section{Authors' contributions}

Conceptualization, AJ, VS and DB; methodology, AJ, VS and TS; software, AJ, TS; validation, AJ, VS, TS and DB; formal analysis, AJ; investigation, AJ, TS and VS; writing —original draft preparation, AJ and TS; writing — review and editing, VS, CBS, MH, RS and DB; visualization, AJ; supervision, VS and DB; project administration, RS and DB; funding acquisition, RS and DB. All authors read and approved the final manuscript.

\section{Author details}

${ }^{1}$ Institute of Electrical and Biomedical Engineering, UMIT-Private University for Health Sciences, Medical Informatics and Technology, Eduard-Wallnöfer-Zentrum 1,6060 Hall in Tirol, Austria. ${ }^{2}$ Leibniz Institute of Photonic Technology (Leibniz IPHT), Albert-Einstein-Straße 9, 07745 Jena, Germany. ${ }^{3}$ Institute of Biomedical Engineering and Informatics, Technische Universität IImenau, Max-Planck-Ring 14, 98693 IImenau, Germany.

\section{Publisher's Note}

Springer Nature remains neutral with regard to jurisdictional claims in published maps and institutional affiliations.

Received: 28 December 2019 Accepted: 9 September 2020 Published online: 16 September 2020

\section{References}

1. Richter H, Kettering M, Wiekhorst F, Steinhoff U, Hilger I, Trahms L. Magnetorelaxometry for localization and quantification of magnetic nanoparticles for thermal ablation studies. Phys Med Biol. 2010;55(3):623.

2. Wiekhorst F, Steinhoff U, Eberbeck D, Trahms L. Magnetorelaxometry assisting biomedical applications of magnetic nanoparticles. Pharm Res. 2012;29(5):1189-202.

3. Chantrell R, Hoon S, Tanner BK. Time-dependent magnetization in fine-particle ferromagnetic systems. J Magn Magn Mater. 1983;38:133-41.

4. Johnson C, Adolphi NL, Butler KL, Lovato DM, Larson R, Schwindt PD, Flynn ER. Magnetic relaxometry with an atomic magnetometer and SQUID sensors on targeted cancer cells. J Magn Magn Mater. 2012;324(17):2613-9.

5. Weis A, Colombo S, Dolgovskiy V, Grujić Z, Lebedev V, Zhang J. Characterizing and imaging magnetic nanoparticles by optical magnetometry. J Phys Conf Ser. 2017;793:012032.

6. Baffa O, Matsuda R, Arsalani S, Prospero A, Miranda J, Wakai R. Development of an optical pumped gradiometric system to detect magnetic relaxation of magnetic nanoparticles. J Magn Magn Mater. 2019;475:533-8.

7. Ludwig F, Mäuselein S, Heim E, Schilling M. Magnetorelaxometry of magnetic nanoparticles in magnetically unshielded environment utilizing a differential fluxgate arrangement. Rev Sci Instrum. 2005;76(10):106102.

8. Liebl M, Wiekhorst F, Eberbeck D, Radon P, Gutkelch D, Baumgarten D, Steinhoff U, Trahms L. Magnetorelaxometry procedures for quantitative imaging and characterization of magnetic nanoparticles in biomedical applications. Biomed Tech. 2015;60(5):427-43.

9. Romanus E, Berkov D, Prass S, Groß C, Weitschies W, Weber P. Determination of energy barrier distributions of magnetic nanoparticles by temperature dependent magnetorelaxometry. Nanotechnology. 2003;14(12):1251.

10. Liebl M, Steinhoff U, Wiekhorst F, Haueisen J, Trahms L. Quantitative imaging of magnetic nanoparticles by magnetorelaxometry with multiple excitation coils. Phys Med Biol. 2014;59(21):6607.

11. Jaufenthaler A, Schier P, Middelmann T, Liebl M, Wiekhorst F, Baumgarten D. Quantitative 2D magnetorelaxometry imaging of magnetic nanoparticles using optically pumped magnetometers. Sensors. 2020;20(3):753. 
12. Warzemann L, Schambach J, Weber P, Weitschies W, Kötitz R. LTS SQUID gradiometer system for in vivo magnetorelaxometry. Supercond Sci Technol. 1999;12(11):953.

13. Dolgovskiy V, Lebedev V, Colombo S, Weis A, Michen B, Ackermann-Hirschi L, Petri-Fink A. A quantitative study of particle size effects in the magnetorelaxometry of magnetic nanoparticles using atomic magnetometry. J Magn Magn Mater. 2015;379:137-50.

14. Shah V, Knappe S, Schwindt PD, Kitching J. Subpicotesla atomic magnetometry with a microfabricated vapour cell. Nat Photonics. 2007;1(11):649.

15. Maser D, Pandey S, Ring H, Ledbetter MP, Knappe S, Kitching J, Budker D. Note: Detection of a single cobalt microparticle with a microfabricated atomic magnetometer. Rev Sci Instrum. 2011;82(8):086112.

16. Scholtes T, Schultze V, IJsselsteijn R, Woetzel S, Meyer H-G. Light-narrowed optically pumped Mx magnetometer with a miniaturized Cs cell. Phys Rev A. 2011:84(4):043416.

17. Schultze V, Scholtes T, IJsselsteijn R, Meyer H-G. Improving the sensitivity of optically pumped magnetometers by hyperfine repumping. J Opt Soc Am B. 2015;32(5):730-6.

18. Schultze V, Schillig B, IJsselsteijn R, Scholtes T, Woetzel S, Stolz R. An optically pumped magnetometer working in the light-shift dispersed Mz mode. Sensors. 2017;17(3):561.

19. Colombo S, Lebedev V, Grujic ZD, Dolgovskiy V, Weis A. MPS and ACS with an atomic magnetometer. Int J Magn Part Imaging. 2016;2(1):606002

20. Colombo S, Lebedev VN, Tonyushkin A, Grujic ZD, Dolgovskiy V, Weis A. Towards a mechanical MPI scanner based on atomic magnetometry. Int J Magn Part Imaging. 2017;3(1):703006.

21. Sarangi S, Tan I-C, Brazdeikis A. Magnetic imaging method based on magnetic relaxation of magnetic nanoparticles J Appl Phys. 2009;105:093926.

22. Rusakov V, Raikher Y. Magnetorelaxometry in the presence of a DC bias field of ferromagnetic nanoparticles bearing a viscoelastic corona. Sensors. 2018:18:1661.

23. Kötitz R, Fannin P, Trahms L. Time-domain study of Brownian and Néel relaxation in ferrofluids. J Magn Magn Mater 1995;149:42-6.

24. Deissler RJ, Wu Y, Martens MA. Dependence of Brownian and Néel relaxation times on magnetic field strength. Med Phys. 2014;41(1):012301.

25. Eberbeck D, Hartwig S, Steinhoff U, Trahms L. Description of the magnetization decay in ferrofluids with a narrow particle size distribution. Magnetohydrodynamics. 2003;39(1):77-83.

26. Néel L. Théorie du traînage magnétique des ferromagnétiques en grains fins avec applications aux terres cuites. Ann Geophys. 1949;5:99-136

27. Schultze V, IJsselsteijn R, Meyer H-G. Noise reduction in optically pumped magnetometer assemblies. Appl Phys B, Lasers Opt. 2010;100:717-24

28. Woetzel S, Schultze V, IJsselsteijn R, Schulz T, Anders S, Stolz R, Meyer H-G. Microfabricated atomic vapor cell arrays for magnetic field measurements. Rev Sci Instrum. 2011;82:033111.

29. Bell WE, Bloom AL. Optically driven spin precession. Phys Rev Lett. 1961;6(6):280.

30. Schultze V, IJsselsteijn R, Scholtes T, Woetzel S, Meyer H-G. Characteristics and performance of an intensity-modulated optically pumped magnetometer in comparison to the classical Mx magnetometer. Opt Express. 2012:20(13):14201-12

31. Hedayati M, Abubaker-Sharif B, Khattab M, Razavi A, Mohammed I, Nejad A, Wabler M, Zhou H, Mihalic J, Gruettner C et al. An optimised spectrophotometric assay for convenient and accurate quantitation of intracellular iron from iron oxide nanoparticles. Int J Hyperth. 2018;34(4):373-81.

32. Eberbeck D, Wiekhorst F, Steinhoff U, Trahms L. Aggregation behaviour of magnetic nanoparticle suspensions investigated by magnetorelaxometry. J Phys Condens Matter. 2006:18:2829-46.

33. Garanin D. Integral relaxation time of single-domain ferromagnetic particles. Phys Rev E. 1996;54(4):3250.

34. Coffey WT, Crothers D, Kalmykov YP, Massawe ES, Waldron J. Exact analytic formula for the correlation time of a single-domain ferromagnetic particle. Phys Rev E. 1994;49(3):1869.

35. Byrd RH, Schnabel RB, Shultz GA. Approximate solution of the trust region problem by minimization over two-dimensional subspaces. Math Program. 1988:40(1-3):247-63.

36. Schwindt $P$, Hollberg L, Kitching J. Self-oscillating rubidium magnetometer using nonlinear magneto-optical rotation. Rev Sci Instrum. 2005:76(12):126103.

37. Sarangi S, Tan I, Brazdeikis A. Brownian relaxation of interacting magnetic nanoparticles in a colloid subjected to a pulsatile magnetic field. J Nanosci Nanotechnol. 2011:11(5):4136-41.

38. Coffey W, Crothers D, Kalmykov YP, Waldron J. Constant-magnetic-field effect in Néel relaxation of single-domain ferromagnetic particles. Phys Rev B. 1995;51(22):15947.

39. Shliomis M, Stepanov V. Theory of the dynamic susceptibility of magnetic fluids. Adv Chem Phys Relax Phenom Condens Matter. 1994;87:1-30

40. Dieckhoff J, Eberbeck D, Schilling M, Ludwig F. Magnetic-field dependence of Brownian and Néel relaxation times. J Appl Phys. 2016;119(4):043903.

41. Pankhurst QA, Connolly J, Jones SK, Dobson J. Applications of magnetic nanoparticles in biomedicine. J Phys D, Appl Phys. 2003;36(13):167.

42. Coene A, Leliaert J, Liebl M, Loewa N, Steinhoff U, Crevecoeur G, Dupré L, Wiekhorst F. Multi-color magnetic nanoparticle imaging using magnetorelaxometry. Phys Med Biol. 2017:62(8):3139.

43. Crevecoeur G, Baumgarten D, Steinhoff U, Haueisen J, Trahms L, Dupré L. Advancements in magnetic nanoparticle reconstruction using sequential activation of excitation coil arrays using magnetorelaxometry. IEEE Trans Magn. 2012;48(4):1313-6.

44. Wilson N, Li R, Perrella C, Light P, Anderson R, Luiten A. A high-bandwidth atomic magnetometer. In: AOS Australian conference on optical fibre technology (ACOFT) and Australian conference on optics, lasers, and spectroscopy (ACOLS) 2019. vol. 11200. Bellingham: International Society for Optics and Photonics; 2019. p. 112000.

45. Sheng D, Li S, Dural N, Romalis MV. Subfemtotesla scalar atomic magnetometry using multipass cells. Phys Rev Lett. 2013;110(16):160802.

46. Limes M, Foley E, Kornack T, Caliga S, McBride S, Braun A, Lee W, Lucivero V, Romalis M. Total-field atomic gradiometer for unshielded portable magnetoencephalography. 2020. arXiv preprint. arXiv:2001.03534. 